\title{
Vehicle Active Steering Control System Based on Human Mechanical Impedance Properties of the Arms
}

\author{
Yoshiyuki Tanaka, Member, IEEE, Naoki Yamada, Toshio Tsuji, Member, IEEE, and Takamasa Suetomi
}

\begin{abstract}
This paper presents the experimental data of human mechanical impedance properties (HMIP) of the arms measured in the steering operations according to the angle of a steering wheel (limbs posture) and the steering torque (muscle co-contraction). The HMIP data shows that human stiffness/viscosity has the minimum/maximum value at the neutral angle of the steering wheel in relax (standard condition) and increases/decreases for the amplitude of steering angle and torque, and that the stability of arms motion in handling the steering wheel becomes high around the standard condition. Next a novel methodology for designing an adaptive steering control system based on HMIP of the arms is proposed, and the effectiveness was then demonstrated via a set of double-lanechange tests with several subjects using the originally developed stationary driving simulator and the 4-DOF driving simulator with a movable cockpit.
\end{abstract}

Index Terms-Human mechanical impedance, active steering control, steering operation, vehicle

\section{INTRODUCTION}

$\mathbf{H}$ UMAN drivers skillfully maneuver a steering wheel by the arms in vehicle driving, in which they actively adjust the conditions of the neuromuscular system (NMS), such as limbs posture and muscle activation, to enable the appropriate control of vehicle motion for driving situations. It is naturally required to consider such human factors in the development of a steering control system so that the driving performance and safety would be further improved.

The recent progress of electric-power-assist steering (EPAS) devises as well as the steer-by-wire (SBW) technology enables to adapt steering operational loads to specific driving conditions [1], [2], and supports to embed human factors into the steering control system. It brings a numbers of researches on the intelligent steering control system including NMS dynamics corresponding to dynamic properties of driver's arms.

Generally dynamic properties of human limb can be expressed using mechanical impedance parameters, i.e., stiffness, viscosity, and inertia, and many experimental studies on human hand impedance have been reported. Mussa-Ivaldi et al. [3]

Y. Tanaka is with Graduate School of Engineering, Nagasaki University, 1-14 Bunkyou-machi, Nagasaki 852-8521, Japan (e-mail: ytnk@ nagasakiu.ac.jp).

N. Yamada and T. Suetomi are with Mazda Motor Corporation, 3-1 Shinchi, Fuchu-cho, Aki-gun, Hiroshima 730-8670, Japan (yamada.na@mazda.co.jp, suetomi.t@mazda.co.jp).

T. Tsuji is with Graduate of Engineering, Hiroshima University, 1-4-1 Kagamiyama, Higashi-hiroshima 739-8527, Japan (email:tsuji@bsys.hiroshima-u.ac.jp). pioneered the measurement of human hand impedance and examined hand stiffness within a stable arm posture. They found that stiffness strongly depends on the arm posture. Dolan et al. [4] and Tsuji et al. [5] also showed that human hand viscoelasticity is considerably affected by muscle activation levels during isometric contraction. Lacquaniti et al. [6] and Gomi and Kawato [7] reported that human hand stiffness greatly changed in arm movements for a manual task. These experimental studies reveal how humans control mechanical impedance of the limbs by regulating the conditions of the NMS.

Some studies on human mechanical impedance during steering operation by the arms have also been reported [8][16]. For example, Bajcinca et al. [11] designed the robust control system including a model of NMS dynamics for SBW systems, and suggested that human stiffness has an important role for the robust stability for uncertain physical parameters in the steering control system through simulation and experimental tests under the limited conditions. However, they treated human viscosity as a constant in their control system. Pick and Cole [12] measured driver's mechanical impedance at holding a steering wheel according to the magnitude of steering torque and muscle co-contraction level, where two hands were arranged at the quarter to three position and the arms were maintained for the straight-driving situation. They [13] developed the driver model with LQR path-following controller and NMS to analyze the robustness of driver steering control with measured mechanical impedance parameters, and demonstrated the differences of pass-following performance between the relax condition and the co-contraction stiffness of NMS. Abbink and Mulder et al. [14], [15] estimated the admittance of NMS in the manual control task by analyzing involuntary limb motion of the driver caused by vehicle accelerations, called biodynamic feedthrough (BDFT), in the limited experimental conditions on muscle co-contraction level. They described the BDFT system composed of the transfer function from body acceleration to involuntary force and the function from force to position. Ikeura et al. [16] reported the close relationship between the hand stiffness measured experimentally during steering operations by the arms and the steering operational feeling using the original driving simulator. These previous researches strongly support the importance of human mechanical impedance properties (HMIP) of the arms in steering for developing an advanced steering control system, but there exists no report of HMIP with significant experimental data. 
On the other hand, a driver-steering system constructed with impedance-controlled robotic devices can be regarded as one of human-machine systems. Many methods have been proposed for designing and controlling a human-machine system constructed with an impedance-controlled robot [17]-[26] since the overall system can be described by the impedance property. Those studies can be classified into two types according to whether the human impedance property is constant during operation [18], [19], or variable [20], [21], [22], [23]. Some research studies addressed the method for providing the realistic feel to improve performances in the manual task [20], [25], [26], but no detailed discussion has addressed how a machine should adapt its dynamics for HMIP that would change widely depending on the NMS condition during manual operations.

The purposes in this research paper are to present 1) the effective data of HMIP during the steering operation by the arms, and 2) a novel methodology for designing an active steering control system based on HMIP by expanding the conventional researches for human-machine systems.

The rest part of this paper is organized as follows: Section II describes the experimental setup developed using robotic devices to measure HMIP in steering operations by the arms. Section III explains a steering control method that automatically adapts mechanical properties of the variable-impedance controlled steering device according to human arms motions and vehicle behaviors. Finally, Section IV demonstrates the effectiveness of the proposed steering control system via the double-lane-change test using the originally developed stationary driving simulator and the 4-DOF driving simulator.

\section{Human Mechanical Impedance Properties of THE ARMS IN STEERING OPERATION}

\section{A. Measurement Method of Human Impedance Properties}

Human hand movements during steering operations are restricted within the rotational axis of the steering wheel with radius $r$ as shown in Fig. 1, and dynamic characteristics of the hand around the steering rotational axis can be expressed with an impedance model [3] as

$$
M_{\theta h} \ddot{\theta}(t)+B_{\theta h} \dot{\theta}(t)+K_{\theta h}\left(\theta(t)-\theta_{c}(t)\right)=-\tau_{e}(t),
$$

where $\tau_{e}$ is the steering torque generated by the operator; $\theta$ the steering angle; $\theta_{c}$ a virtual trajectory of hand equilibrium; $M_{\theta h}, B_{\theta h}, K_{\theta h}$ represent hand inertia, viscosity, and stiffness around the rotational axis of the steering. Assuming $\theta_{c}$ and human impedance parameters $\left(M_{\theta h}, B_{\theta h}, K_{\theta h}\right)$ were constant for a short duration, Eq. (1) could be rewritten as follows:

$$
M_{\theta h} \ddot{\theta}(t)+B_{\theta h} \dot{\theta}(t)+K_{\theta h}\left(\theta(t)-\theta_{c}\right)=-\tau_{e}(t) .
$$

At the onset time of the external disturbance $t_{0}$, we have

$$
M_{\theta h} \ddot{\theta}\left(t_{0}\right)+B_{\theta h} \dot{\theta}\left(t_{0}\right)+K_{\theta h}\left(\theta\left(t_{0}\right)-\theta_{c}\right)=-\tau_{e}\left(t_{0}\right),
$$

and $\theta_{c}$ can be eliminated from Eqs. (2) and (3) as

$$
M_{\theta h} d \ddot{\theta}(t)+B_{\theta h} d \dot{\theta}(t)+K_{\theta h} d \theta(t)=-d \tau(t),
$$

where $d \theta(t) \equiv \theta(t)-\theta\left(t_{0}\right), d \tau(t) \equiv \tau_{e}(t)-\tau_{e}\left(t_{0}\right)$. Thus, the human impedance parameters can be estimated by fitting

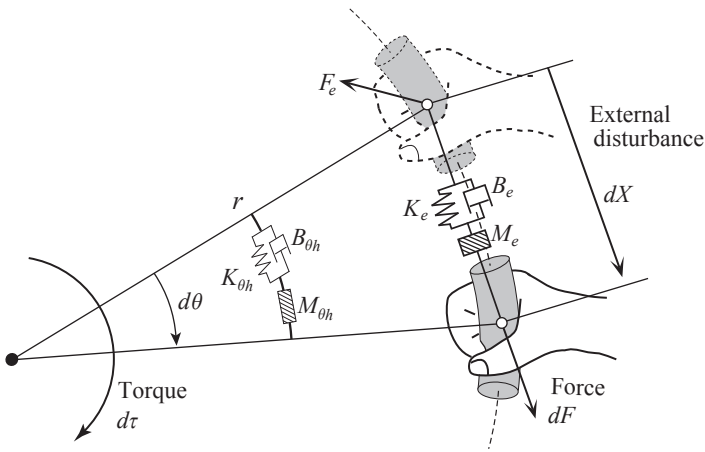

Fig. 1. Schematic description of human impedance measurement during steering operation.

the measured time sequences of steering angle and torque for the external disturbance of short duration into Eq. (4) with the least squares method.

On the other hand, a hand displacement $d X$ corresponding to $d \theta$ can be given by

$$
d X(t) \approx r d \theta(t)
$$

and a reaction force to his hand $d F$ is

$$
d F(t)=\frac{1}{r} d \tau(t)
$$

Accordingly, dynamic characteristics of the human hand in the tangential direction of the steering wheel can be expressed as

$$
M_{e} d \ddot{X}(t)+B_{e} d \dot{X}(t)+K_{e} d X(t)=-d F(t),
$$

where hand impedance parameters in the tangential direction are calculated by

$$
M_{e}=\frac{1}{r^{2}} M_{\theta h}, B_{e}=\frac{1}{r^{2}} B_{\theta h}, K_{e}=\frac{1}{r^{2}} K_{\theta h} .
$$

\section{B. Experimental Apparatus}

Fig. 2 shows a schematic overview of the stationary driving simulator developed for this paper. The driving simulator is constructed with a direct-drive-type motor (NSK, Ltd., maximum torque: $20 \mathrm{Nm}$ ), a computer for controlling the motor, and two LCD displays to monitor steering angle and torque measured (Display I) and for the present of a driving scene created with a real-time 3-D animation software (Display II). A steering wheel (NARDI, Ltd., radius: $r=0.185 \mathrm{~m}$ ) and a rotation torque sensor (SOHGOH KEISO Corp., maximum torque: $50 \mathrm{Nm}$ ) are attached to the rotating part of the motor, and the steering angle is measured by an encoder built into the motor (encoder resolution: 51,200 pulse/r). The motor is controlled by a DSP board (dSPACE: ds1103) that can provide stable control and high-quality data measurement at $2 \mathrm{kHz}$ sampling-frequency.

Fig. 3 shows an example of the steering angle $d \theta$, the angular velocity $d \dot{\theta}$, the angular acceleration $d \ddot{\theta}$ and the torque $d \tau$ measured in estimating mechanical impedance properties of the known spring-mass system $\left(M_{e}=0.5 \mathrm{~kg}, K_{e}=879\right.$ $\mathrm{N} / \mathrm{m}$ ) which was attached to the steering wheel. Mean values and standard deviation for five sets of estimated results are 


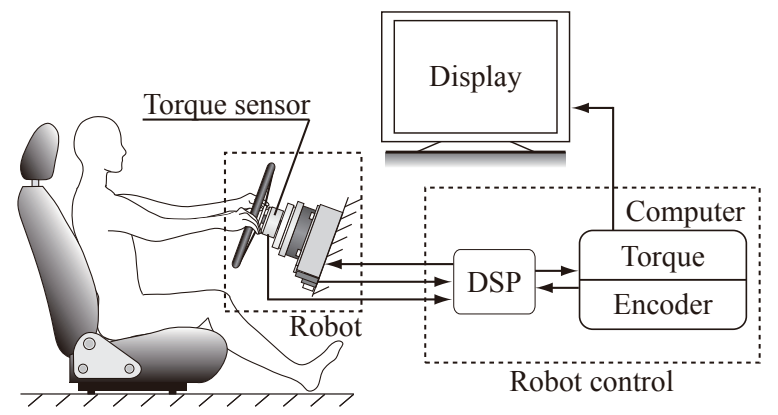

(a)

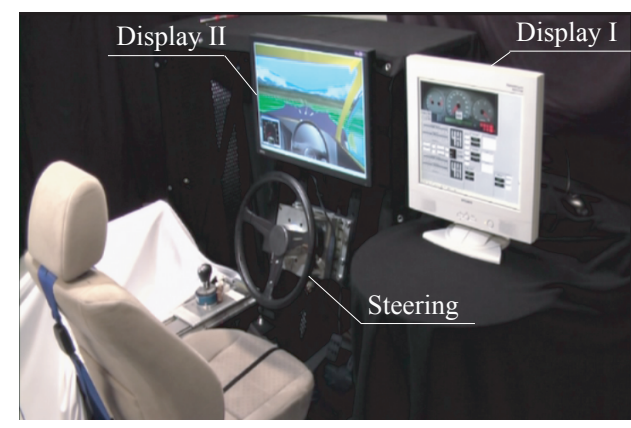

(b)

Fig. 2. An overview of the developed stationary driving simulator.

$M_{e}=0.52 \pm 0.01 \mathrm{~kg}$ and $K_{e}=874.9 \pm 28.6 \mathrm{~N} / \mathrm{m}$, respectively. In the bottom figure, the solid line is the measured torque and the broken line is the calculated torque using Eq. (4) while the estimated $M_{\theta h}, B_{\theta h}$, and $K_{\theta h}$. The results demonstrate that the developed driving system can estimate impedance properties successfully.

\section{Experimental method}

Fig. 4 represents the experimental condition designed in this study. A human subject sat in front of the experimental system, and his shoulders were restrained to the seat back using a seatbelt. Both hands were fixed to the steering wheel using a plastic cast to eliminate the passive impedance of the hand palm. The subject was asked to generate the specified steering torque while maintaining arm posture using the biofeedback display that provides the magnitude of steering torque by the bar graph as shown in Fig. 4(b). In the measurement of human mechanical impedance properties (HMIP) in operating the steering wheel by the arms, a dual-arm grip position producing a symmetrical appearance was set as the position of the right hand $\theta_{d}=0, \pi / 6, \pi / 3, \ldots, 2 \pi / 3 \mathrm{rad}$, and the magnitude of the target steering torque $\tau_{d}=-9,-6, \ldots, 0, \ldots, 6,9 \mathrm{Nm}$. The number of trials was ten for each condition.

Six healthy subjects (male university students aged 2224 years old) participated in the measurement experiment of HMIP, who were interested in this automobile research.

\section{Experimental results}

Table I summarizes the experimental data of HMIP of the arms around the steering rotational axis depending on the specified steering angle $\theta_{d}$ and torque $\tau_{d}$, where the mean
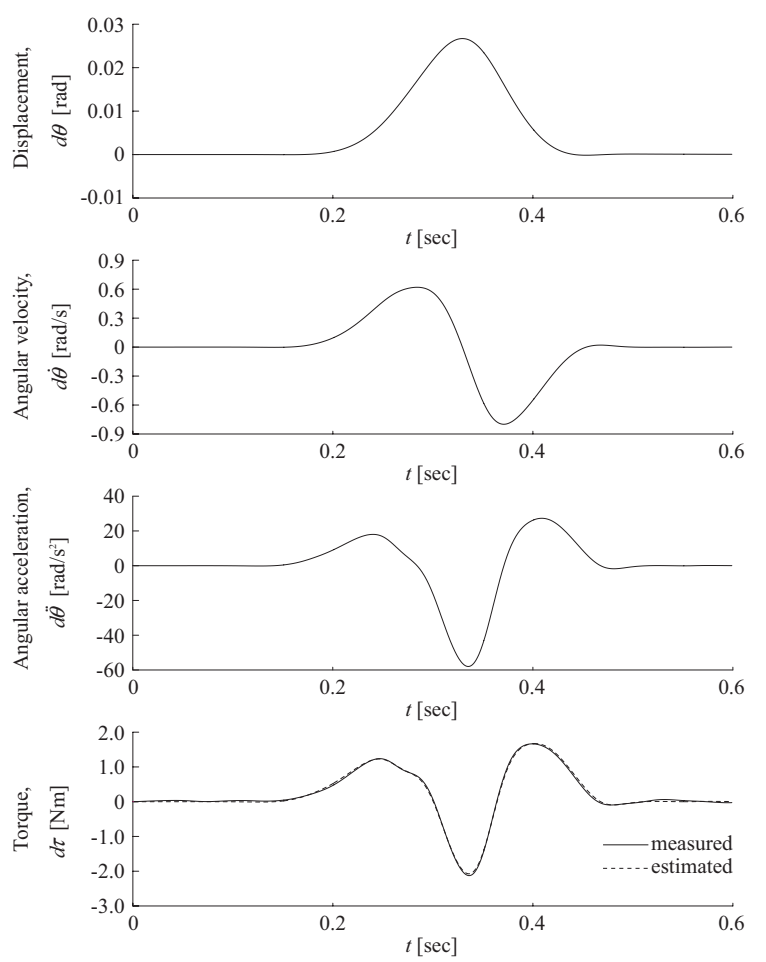

Fig. 3. An example of the measured signals for estimating known mechanical impedance properties.

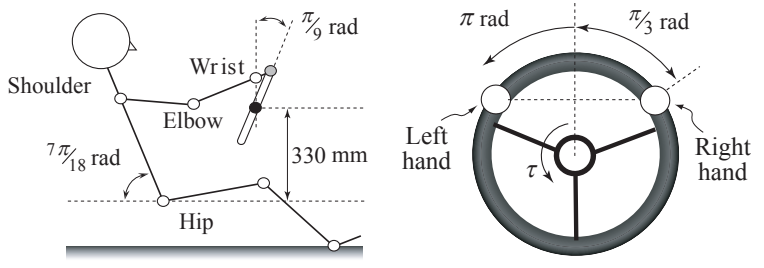

(a)

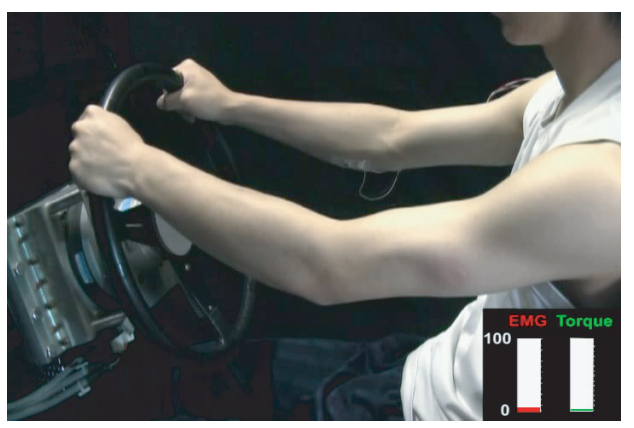

(b)

Fig. 4. Experimental condition for the measurements of human impedance properties in steering by the arms.

values and standard deviations of the measured results for all subjects are presented. Note that each of HMIPs was estimated using Eq. (4) with the time sequences of steering angle and steering torque measured during the external disturbance of short duration.

Fig. 5 visualizes the changes of HMIP in $\theta_{d}$ and $\tau_{d}$ using the mean values calculated using the measured data for all subjects given in Table I by a color map, where human stiffness, 
TABLE I

MEAN VALUES AND STANDARD DEVIATIONS OF THE MEASURED HMIP OF THE ARMS AROUND THE ROTATIONAL AXIS OF THE STEERING WHEEL DEPENDING ON STEERING ANGLE AND TORQUE.

\begin{tabular}{|c|c|c|c|c|c|c|c|c|}
\hline & $\theta_{d} \mid[\mathrm{rad}]$ & $\tau_{d}=-9 \mathrm{Nm}$ & $\tau_{d}=-6 \mathrm{Nm}$ & $\tau_{d}=-3 \mathrm{Nm}$ & $\tau_{d}=0 \mathrm{Nm}$ & $\tau_{d}=3 \mathrm{Nm}$ & $\tau_{d}=6 \mathrm{Nm}$ & $\tau_{d}=9 \mathrm{Nm}$ \\
\hline \multirow{5}{*}{$\begin{array}{c}\text { Stiffness, } K_{\theta h} \\
{[\mathrm{Nm} / \mathrm{rad}]}\end{array}$} & 0 & 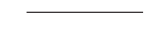 & - & 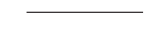 & $10.13 \pm 4.686$ & $16.42 \pm 4.279$ & $27.06 \pm 5.531$ & $35.94 \pm 4.175$ \\
\hline & $\pi / 6$ & $33.43 \pm 5.437$ & $25.39 \pm 5.333$ & $12.89 \pm 6.978$ & $7.99 \pm 3.326$ & $11.34 \pm 5.134$ & $24.42 \pm 9.703$ & $35.42 \pm 10.112$ \\
\hline & $\pi / 3$ & $34.85 \pm 4.223$ & $32.06 \pm 6.777$ & $20.74 \pm 7.229$ & $10.97 \pm 7.090$ & $15.77 \pm 6.694$ & $25.97 \pm 6.714$ & $34.47 \pm 10.543$ \\
\hline & $\pi / 2$ & $45.06 \pm 8.264$ & $41.06 \pm 2.146$ & $25.91 \pm 6.136$ & $17.13 \pm 5.690$ & $16.17 \pm 7.394$ & $26.82 \pm 5.181$ & $36.90 \pm 6.675$ \\
\hline & $2 \pi / 3$ & $52.06 \pm 15.329$ & $44.63 \pm 11.139$ & $37.32 \pm 9.691$ & $29.83 \pm 11.541$ & $29.11 \pm 11.977$ & $39.68 \pm 9.987$ & $46.78 \pm 11.180$ \\
\hline \multirow{5}{*}{$\begin{array}{c}\text { Viscosity, } B_{\theta h} \\
{[\mathrm{Nms} / \mathrm{rad}]}\end{array}$} & 0 & $\longrightarrow$ & - & - & $1.84 \pm 0.397$ & $1.81 \pm 0.371$ & $1.49 \pm 0.401$ & $1.20 \pm 0.323$ \\
\hline & $\pi / 6$ & $1.04 \pm 0.304$ & $1.26 \pm 0.311$ & $1.67 \pm 0.392$ & $1.86 \pm 0.412$ & $1.89 \pm 0.331$ & $1.60 \pm 0.375$ & $1.26 \pm 0.332$ \\
\hline & $\pi / 3$ & $1.11 \pm 0.206$ & $1.19 \pm 0.250$ & $1.51 \pm 0.229$ & $1.51 \pm 0.338$ & $1.67 \pm 0.316$ & $1.53 \pm 0.306$ & $1.34 \pm 0.402$ \\
\hline & $\pi / 2$ & $1.06 \pm 0.352$ & $1.14 \pm 0.345$ & $1.36 \pm 0.363$ & $1.36 \pm 0.447$ & $1.45 \pm 0.539$ & $1.42 \pm 0.466$ & $1.22 \pm 0.488$ \\
\hline & $2 \pi / 3$ & $1.24 \pm 0.217$ & $1.24 \pm 0.206$ & $1.30 \pm 0.338$ & $1.35 \pm 0.368$ & $1.46 \pm 0.441$ & $1.24 \pm 0.335$ & $0.98 \pm 0.210$ \\
\hline \multirow{5}{*}{$\begin{array}{c}\text { Inertia, } M_{\theta h} \\
{\left[\mathrm{kgm}^{2} \times 10^{-2}\right]}\end{array}$} & 0 & $\longrightarrow$ & $\longrightarrow$ & 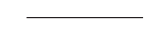 & $12.62 \pm 0.015$ & $12.90 \pm 0.018$ & $13.60 \pm 0.014$ & $14.02 \pm 0.013$ \\
\hline & $\pi / 6$ & $13.07 \pm 0.014$ & $12.67 \pm 0.013$ & $11.89 \pm 0.013$ & $11.67 \pm 0.015$ & $11.69 \pm 0.017$ & $12.47 \pm 0.019$ & $12.77 \pm 0.015$ \\
\hline & $\pi / 3$ & $11.05 \pm 0.007$ & $10.81 \pm 0.007$ & $10.10 \pm 0.007$ & $9.82 \pm 0.011$ & $9.64 \pm 0.012$ & $10.15 \pm 0.006$ & $10.45 \pm 0.004$ \\
\hline & $\pi / 2$ & $9.40 \pm 0.013$ & $9.03 \pm 0.010$ & $8.41 \pm 0.011$ & $8.58 \pm 0.012$ & $8.24 \pm 0.009$ & $8.77 \pm 0.009$ & $9.53 \pm 0.012$ \\
\hline & $2 \pi / 3$ & $8.79 \pm 0.013$ & $8.81 \pm 0.014$ & $8.40 \pm 0.013$ & $8.14 \pm 0.012$ & $7.86 \pm 0.012$ & $8.76 \pm 0.014$ & $9.21 \pm 0.014$ \\
\hline
\end{tabular}

viscosity, inertia and the damping coefficient are presented from the top. A white circle is plotted on the measurement conditions, and the data between the white circles is calculated by cubic interpolation based on a Delaunay triangulation.

The maps reveal how HMIP changes in the NMS conditions, i.e., limbs posture $\left(\theta_{d}\right)$ and muscle co-contraction level $\left(\tau_{d}\right)$. Human stiffness $K_{\theta h}$ has the minimum value at the standard condition, the neutral angle of the steering wheel in relax $\left(\theta_{d}\right.$, $\left.\tau_{d}\right)=(0 \mathrm{rad}, 0 \mathrm{Nm})$, and increases for the magnitude of $\theta_{d}$ and $\tau_{d}$. The observed characteristics agrees with the previous studies on human impedance measurement that reported human hand stiffness changes in the limb's posture and increases for the muscle co-contraction level. Interestingly, human viscosity $B_{\theta h}$ has the maximum value at the standard condition, and decrease for the magnitude of $\theta_{d}$ and $\tau_{d}$, although human hand viscosity would increase for the muscle co-contraction level as same as human stiffness addressed in the previous studies. The possible reason is that the measured human viscosity is one around the rotational axis of the steering wheel. While human inertia $M_{\theta h}$ depends on almost the steering angle $\theta_{d}$ that means the limbs posture. Furthermore, the damping coefficient $\zeta_{\theta h}$, the motion stability of the limb in handling the steering wheel, becomes high around the standard condition while decays in the larger magnitude of $\theta_{d}$ and $\tau_{d}$. Paradoxically, it becomes more difficult for human drivers to ensure the stable control of the steering wheel without any assistance from the steering device in the case of driving situations requiring much larger steering angle and torque, such as an emergency obstacle avoidance.

Based on the obtained evidences on HMIP of the arms measured in steering, the next section discusses a methodology to embedded the HMIP depending on the steering angle and torque into an intelligent steering control system assuming EPAS and SBW systems.

\section{Active-Steering Control System Using HMIP}

Fig. 6 (a) shows a block diagram of the proposed steering control system including the database of HMIP of the arms.
The steering motor is under a variable-impedance control, and the database outputs HMIP according to the combination of steering angle $\theta$ and torque $\tau$. Dynamic behaviors of the steering motor around its rotational axis can be given by

$$
M_{\theta s} \ddot{\theta_{v}}+B_{\theta s} \dot{\theta_{v}}+K_{\theta s}\left(\theta_{v}-\theta_{s c}\right)=\tau,
$$

where $M_{\theta s}$ is the steering inertia; $B_{\theta s}$ and $K_{\theta s}$ are the variable viscosity and stiffness, respectively; $\theta_{v}$ is a target angle at the next sampling time calculated from the steering impedance properties with the current steering torque $\tau$; and $\theta_{s c}$ is an equilibrium for $K_{\theta s}$. Note that a function of the damping coefficient of the human-steering system $\zeta$ outputs the steering impedance parameters according to the steering motion in operating.

To simplify the discussion, a human-steering system manipulated by the upper extremities is expressed using a rotational spring-mass-damper system, as shown in Fig. 6 (b). A human driver grasps a steering wheel with inertia of $\left(M_{\theta h}+M_{\theta s}\right)$ and displaces it from the equilibrium with $\theta$. The left spring and dumper work to pull back counterclockwise with $K_{\theta h} \theta$ and $B_{\theta h} \dot{\theta}$ of torque, while the right ones to push back counterclockwise with $K_{\theta s} \theta$ and $B_{\theta s} \dot{\theta}$ of torque. The torque added to the human-steering system $\tau_{e x}$ can be written as follows:

$$
\begin{aligned}
\tau_{e x} & =\tau-\left(K_{\theta h} \theta+K_{\theta s} \theta\right)-\left(B_{\theta h} \dot{\theta}+B_{\theta s} \dot{\theta}\right) \\
& =\tau-\left(K_{\theta h}+K_{\theta s}\right) \theta-\left(B_{\theta h}+B_{\theta s}\right) \dot{\theta} .
\end{aligned}
$$

Thus, the motion equation of the human-steering system can be expressed by

$$
\left(M_{\theta h}+M_{\theta s}\right) \ddot{\theta}+\left(B_{\theta h}+B_{\theta s}\right) \dot{\theta}+\left(K_{\theta h}+K_{\theta s}\right) \theta=\tau .
$$

As dividing both sides of Eq. (11) by $m_{h s} e^{\lambda t}$, we obtain the following equation as

$$
\lambda^{2}+2 \zeta \omega_{n} \lambda+\omega_{n}^{2}=0,
$$

where $\theta=e^{\lambda t}, \zeta=b / 2 \sqrt{m_{h s} k_{h s}}, \omega_{n}=\sqrt{k_{h s} / m_{h s}}, k_{h s}=$ $K_{\theta h}+K_{\theta s}, b_{h s}=B_{\theta h}+B_{\theta s}$, and $m_{h s}=M_{\theta h}+M_{\theta s}$. The 

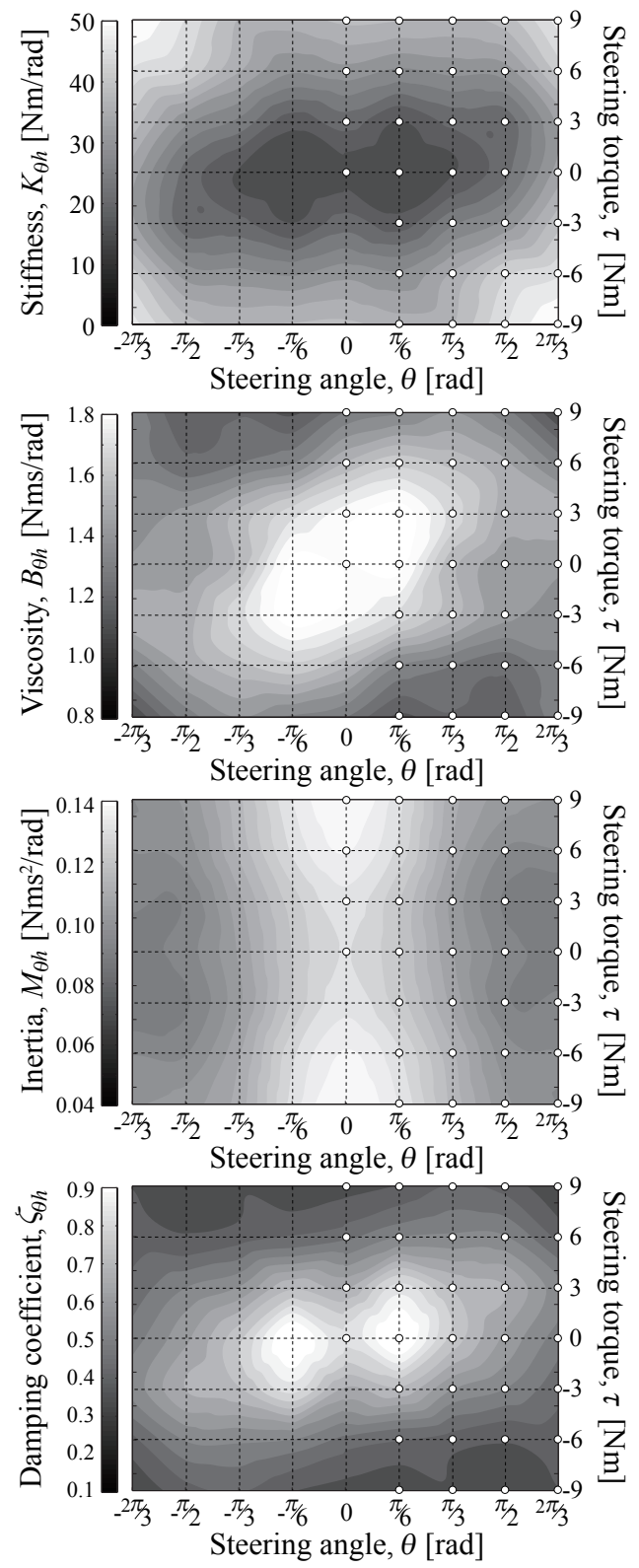

Fig. 5. Dependences of the HMIP of the arms and the damping coefficient on the NMS conditions in the steering operations.

solution of Eq. (12) can be given by

$$
\lambda=-\zeta \omega_{n} \pm \sqrt{\zeta^{2}-1} \omega_{n}
$$

The damping coefficient of the target system $\zeta$ and the natural angular frequency $\omega_{n}$ are calculated by

$$
\begin{gathered}
\zeta=\frac{B_{\theta h}+B_{\theta s}}{2 \sqrt{\left(M_{\theta h}+M_{\theta s}\right)\left(K_{\theta h}+K_{\theta s}\right)}} \\
\omega_{n}=\sqrt{\frac{K_{\theta h}+K_{\theta s}}{M_{\theta h}+M_{\theta s}} .}
\end{gathered}
$$

The driver's load for steering operations increases with the damping coefficient $\zeta$, and vice versa. As $\zeta$ is designed according to the target driving situation, it will be expected that the steering device can adequately assist the driver's steering

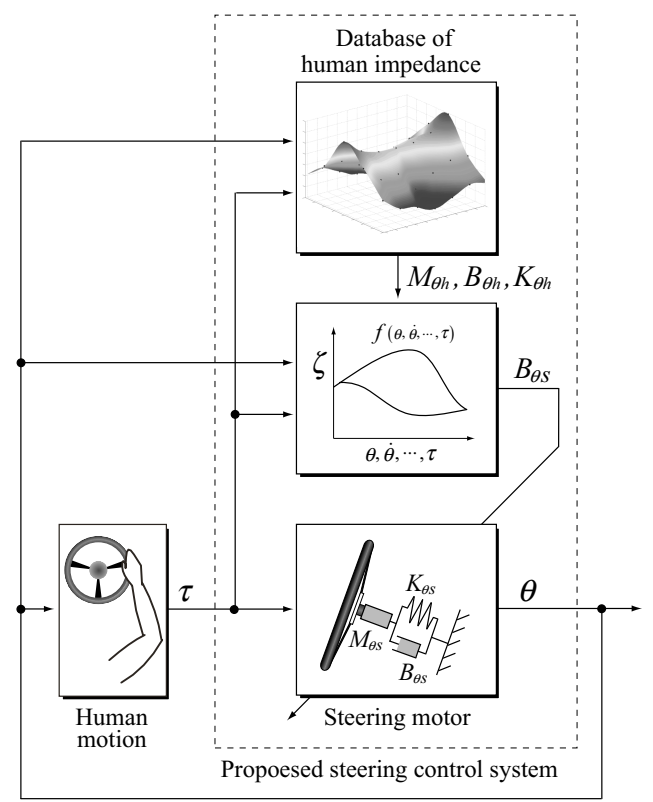

(a)

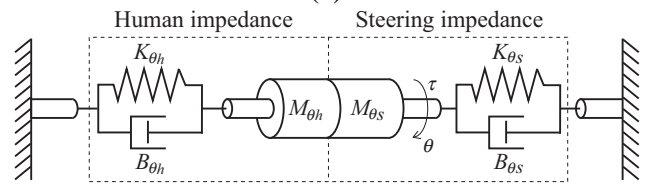

(b)

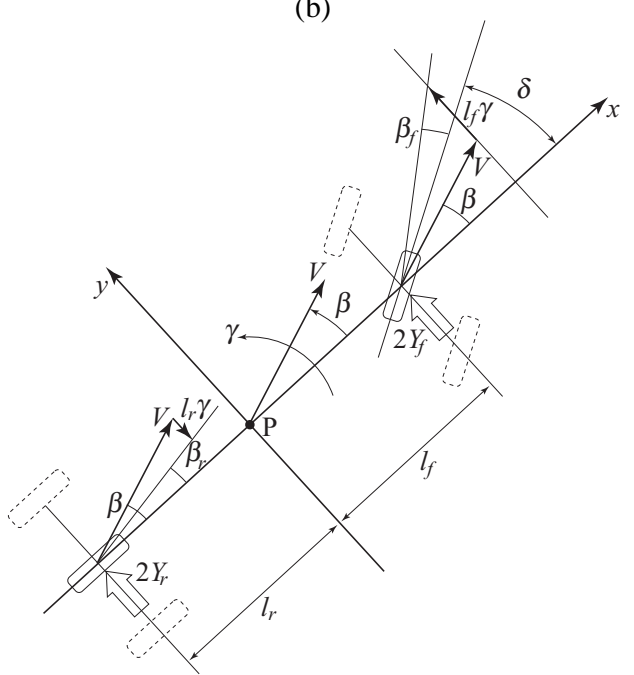

(c)

Fig. 6. Block diagram of the proposed steering control system with considerations of the HMIP database and the vehicle dynamics.

operation by actively regulating its own mechanical dynamics with considerations of HMIP of the arms.

An equivalent two-wheel vehicle model [27] as shown in Fig. 6 (c) was used to represent vehicle dynamics, where the vehicle runs at a specified speed $V$ without sudden acceleration or deceleration. This model considers the lateral and rotational motion of the vehicle's center of gravity as follows:

$$
\begin{gathered}
m V\left(\frac{d \beta}{d t}+\gamma\right)=2 Y_{f}+2 Y_{r} \\
I \frac{d \gamma}{d t}=l_{f} \cdot 2 Y_{f}-l_{r} \cdot 2 Y_{r}
\end{gathered}
$$


with

$$
\begin{aligned}
Y_{f} & =-K_{f}\left(\beta+\frac{l_{f}}{V} \gamma-\delta\right) \\
Y_{r} & =-K_{r}\left(\beta+\frac{l_{r}}{V} \gamma\right),
\end{aligned}
$$

where $m$ is the vehicle's mass, $I$ is the vehicle's moment of inertia, $Y_{f}$ and $Y_{r}$ are the cornering force of the front and rear wheel, $K_{f}$ and $K_{r}$ are the cornering power of the front and rear wheel, $l_{f}$ and $l_{r}$ represent the front and rear wheelbase, the distance between the vehicle's center of gravity and the front or rear axle, $\beta$ is the sideslip angle of the vehicle's center of gravity, $\gamma$ is the yaw angle rate of the vehicle's center of gravity, and $\delta$ is the front wheel angle. The relationship between the steering angle $\theta$ and the front wheel angle $\delta$ with gear ratio $n$ can be expressed by $\delta=\theta / n$. The limits of cornering forces [28] are given by

$$
\begin{aligned}
& Y_{f_{l} \text { imit }}=\mu m g\left(\frac{l_{r}}{l_{f}}+l_{r}\right) \\
& Y_{r_{l} \text { imit }}=\mu m g\left(\frac{l_{f}}{l_{f}+l_{r}}\right),
\end{aligned}
$$

where $\mu$ is the road friction coefficient, and $g$ is the gravitational acceleration. Accordingly the steering stiffness $K_{\theta s}$ representing the steering reaction torque caused by driver's operation and vehicle behavior can be derived as

$$
K_{\theta s}=-\frac{\mu \xi K_{f}}{n^{2}\left(l_{f}+l_{r}\right)}\left(\frac{1}{k_{1}}\left(k_{2} l_{r}-l_{f}\right)-\left(l_{f}+l_{r}\right)\right)
$$

with

$$
\begin{aligned}
k_{1} & =1-\frac{m}{2\left(l_{f}+l_{r}\right)^{2}} \frac{l_{f} K_{f}-l_{r} K_{r}}{K_{f} K_{r}} V^{2} \\
k_{2} & =1-\frac{m}{2\left(l_{f}+l_{r}\right)} \frac{l_{f}}{l_{r} K_{r}} V^{2},
\end{aligned}
$$

where $\xi$ is the trail length.

\section{DRiving Simulator ExPeriments of Double-LANe-Change TASK}

The effectiveness of the proposed steering control system was examined via a double-lane-change (DLC) test, in which a human driver is asked to avoid all cones arranged on the specified test course, as shown in Fig. 7, by quick and precise operations of the steering wheel by the arms. Eight healthy subjects (male university students aged 22-25 years old) participated in the experiments, who were cooperative with this research. Note that Subs. E, F and H drive a car several times a year while the others drive their own car almost every day.

\section{A. Experimental design for DLC tests}

Two test courses were designed based on the ISO guideline [29], and the course parameters were set as in Table II. Course 1 (ISO 3888-1) assumed for a high-speed lane change while Course 2 (ISO 3888-2) for a severe lane change, respectively. In this study, the following four cases were designed as:

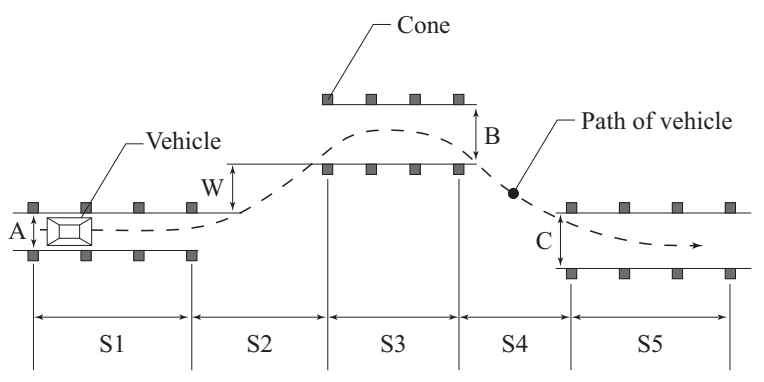

Fig. 7. The regulation course designed for the DLC test (ISO 3888).

TABLE II

LANE PARAMETERS FOR EACH COURSE IN THE DLC TEST [29].

\begin{tabular}{cccccccccc}
\hline & S1 & S2 & S3 & S4 & S5 & A & B & C & W \\
\hline \hline Course 1 & 15 & 30 & 25 & 25 & 30 & $1.1 W+0.25$ & $1.2 W+0.25$ & $1.3 W+0.25$ & $3.5-\mathrm{A}$ \\
Course 2 & 12 & 13.5 & 11 & 12.5 & 12 & $1.1 W+0.25$ & $W+1$ & $1.3 W+0.25$ & 1 \\
\hline
\end{tabular}

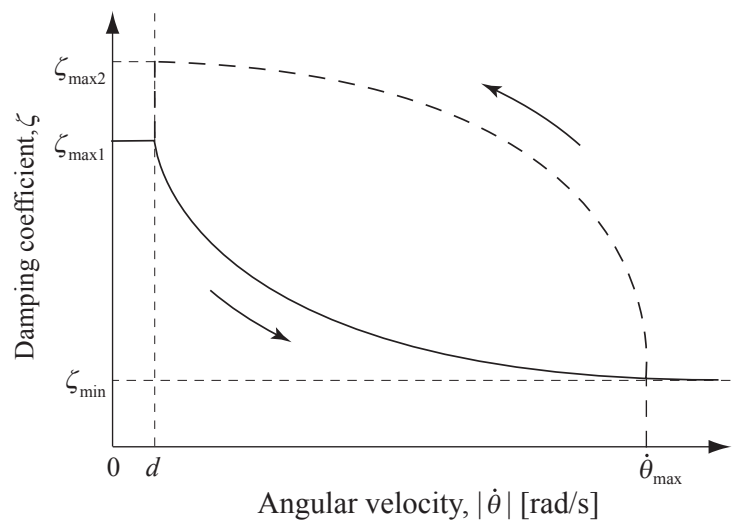

Fig. 8. An overview of the designed regulation function of the damping coefficient of human-vehicle system.

Case Ia: Course 1 with the vehicle speed $80 \mathrm{~km} / \mathrm{h}$

Case Ib: Course 1 with the vehicle speed $100 \mathrm{~km} / \mathrm{h}$

Case IIa: Course 2 with the vehicle speed $40 \mathrm{~km} / \mathrm{h}$

Case IIb: Course 2 with the vehicle speed $60 \mathrm{~km} / \mathrm{h}$

The damping coefficient of the human-steering system $\zeta$ was designed as a function of the absolute value of the steering angular velocity $|\dot{\theta}|$ as shown in Fig. 8, in which $\zeta$ decreases as the velocity increases to enlarge quick handling until its deceleration is occurred and increases to assist the stable positioning of the steering wheel as a driver hoped. The designed regulation function for $\zeta$ is formulated as follows;

$$
\zeta=\left\{\begin{aligned}
& \zeta_{\max 1},(0 \leq|\dot{\theta}|<d) \\
& \frac{b \cdot D C-a_{1}(|\dot{\theta}|-d)}{b+(|\dot{\theta}|-d)}+\zeta_{\min }\left(|\dot{\theta}| \geq d, \frac{d|\dot{\theta}|}{d t} \geq-3 \mathrm{rad} / \mathrm{s}^{2}\right) \\
&-\frac{b \cdot D C-a_{2}\left(-|\dot{\theta}|+d+\dot{\theta}_{\max }\right)}{b+\left(-|\dot{\theta}|+d+\dot{\theta}_{\max }\right)}+\zeta_{\max 2}, \\
&\left(|\dot{\theta}| \geq d, \frac{d|\dot{\theta}|}{d t}<-3 \mathrm{rad} / \mathrm{s}^{2}\right)
\end{aligned}\right.
$$


TABLE III

PARAMETERS OF THE VEHICLE DYNAMICS AND THE PROPOSED STEERING CONTROL SYSTEM UTILIZED IN THE SIMULATOR EXPERIMENTS.

(a)

\begin{tabular}{ll}
\hline Parameter & Set value \\
\hline \hline Vehicle mass, $m$ & $1300 \mathrm{~kg}$ \\
Front wheelbase, $l_{f}$ & $1.2 \mathrm{~m}$ \\
Rear wheelbase, $l_{r}$ & $1.5 \mathrm{~m}$ \\
Yaw inertia moment, $I$ & $2600 \mathrm{kgm}^{2}$ \\
Front cornering power, $K_{f}$ & $40000 \mathrm{~N} / \mathrm{rad}$ \\
Rear cornering power, $K_{r}$ & $40000 \mathrm{~N} / \mathrm{rad}$ \\
Gear ratio, $n$ & 16 \\
Road friction coefficient, $\mu$ & 0.8 \\
Trail length, $\xi$ & $0.04 \mathrm{~m}$ \\
\hline
\end{tabular}

(b)

\begin{tabular}{cccccc}
\hline$\zeta_{\max 2}$ & $\zeta_{\max 1}$ & $\zeta_{\min }$ & $a_{1}$ & $a_{2}$ & $d[\mathrm{rad} / \mathrm{s}]$ \\
\hline 1.0 & 0.98 & 0.3 & 0.6 & 0.01 & 3.0 \\
\hline
\end{tabular}

where $D C=\zeta_{\max }-\zeta_{\min }, b=\frac{\dot{\theta}_{\max }}{D C} \times a, d$ is a dead zone of the angular velocity around $0 \mathrm{rad} / \mathrm{s}, a_{1}$ and $a_{2}$ are parameters that regulate the curvatures of the wave, and $\dot{\theta}_{\max }$ is the value of the angular velocity when switching from acceleration to deceleration.

The DLC tests with the designed four cases were carried out using the proposed steering control system, in which the steering viscosity $B_{\theta s}$ was varied in the rotational velocity of the steering wheel $\dot{\theta}$ by the regulation function $\zeta$ including the measured HMIP of the arms as

$$
B_{\theta s}=2 \zeta \sqrt{\left(K_{\theta s}+K_{\theta h}\right)\left(M_{\theta s}+M_{\theta h}\right)}-B_{\theta h} .
$$

Note that the values of $K_{\theta h}, B_{\theta h}$, and $M_{\theta h}$ are obtained from the HMIP database built into the control structure. Also the standard control system was prepared with fixing the steering viscosity as $B_{\theta s}=1.0 \mathrm{Nms} / \mathrm{rad}$ (CONST), which was determined on the basis of general vehicles. The parameters of the proposed steering control system for the regulation function and the vehicle dynamics were set as Table III.

Ten sets of the DLC test were conducted for each of the four designed cases (i.e., Cases Ia, Ib, IIa, and IIb) with the two steering control systems.

\section{B. Operational Experiment I: DLC test using the stationary driving simulator}

Fig. 9 shows the image examples of the presentation screen for a subject in the DLC tests with the stationary driving simulator shown in Fig. 2. Six subjects (A to F) were participated in the experiment, and conducted ten sets for each driving case.

Fig. 10 (a) and (b) represents the mean values and standard deviations for the success rate of "Avoid", where the subject achieved the first lane change, and the rate of "Return" where he achieved the 1 st and 2 nd lane changes, i.e., DLC. The success rates are improved by the proposed steering control
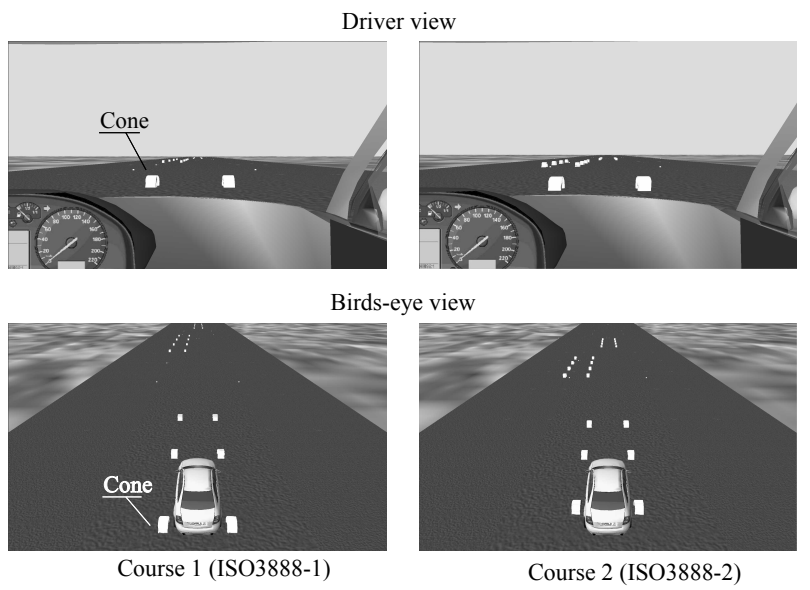

Fig. 9. An example of screen images projected in the DLC test.

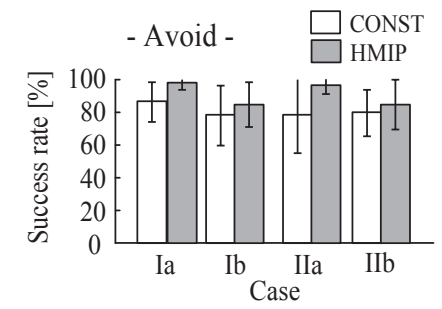

(a)

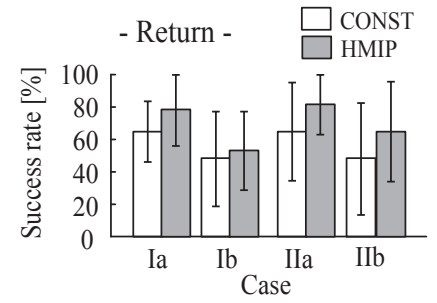

(b)
Fig. 10. Mean success rates and standard deviations for all subjects in each case.

system (HMIP) compared to those with the constant model (CONST), although some individual differences are found.

Fig. 11 shows the typical time histories of steering and vehicle behaviors measured in Case Ia and Case IIa for each type of the two steering control systems. The trajectories of the vehicle's center of gravity, the steering angle $\theta$, the angular velocity $\dot{\theta}$, the steering torque $\tau$, the slip angle of the vehicle $\beta$ and the yaw rate $\gamma$ are plotted from the top. The figure plots the measured waves for ten sets of experiments. The waves are considerably different between HMIP and CONST. Especially, the profiles of the steering angular velocity were sharpened in the proposed human-impedance-based control system.

The operational performance was analyzed by the quantitative index $E$ defined with the operational time $T$ and the trajectory accuracy $J$ defined as:

$$
E=\sqrt{T^{2}+J^{2}}
$$

with

$$
J=\int \varepsilon^{2} d t
$$

where $T$ is the total time during which the steering angular velocity exceeds $0.05 \mathrm{rad} / \mathrm{s}$ and $\varepsilon$ is the lateral deviation from the average trajectory in cases where the subject perfectly avoided all obstacles. Note that smaller values of the indices indicate better performance in the DLC test.

Fig. 12 shows the evaluation results for six subjects, and demonstrates that the $E$ value for the proposed control system was smaller than that for CONST with the significant differences although some individual differences exist. The results 

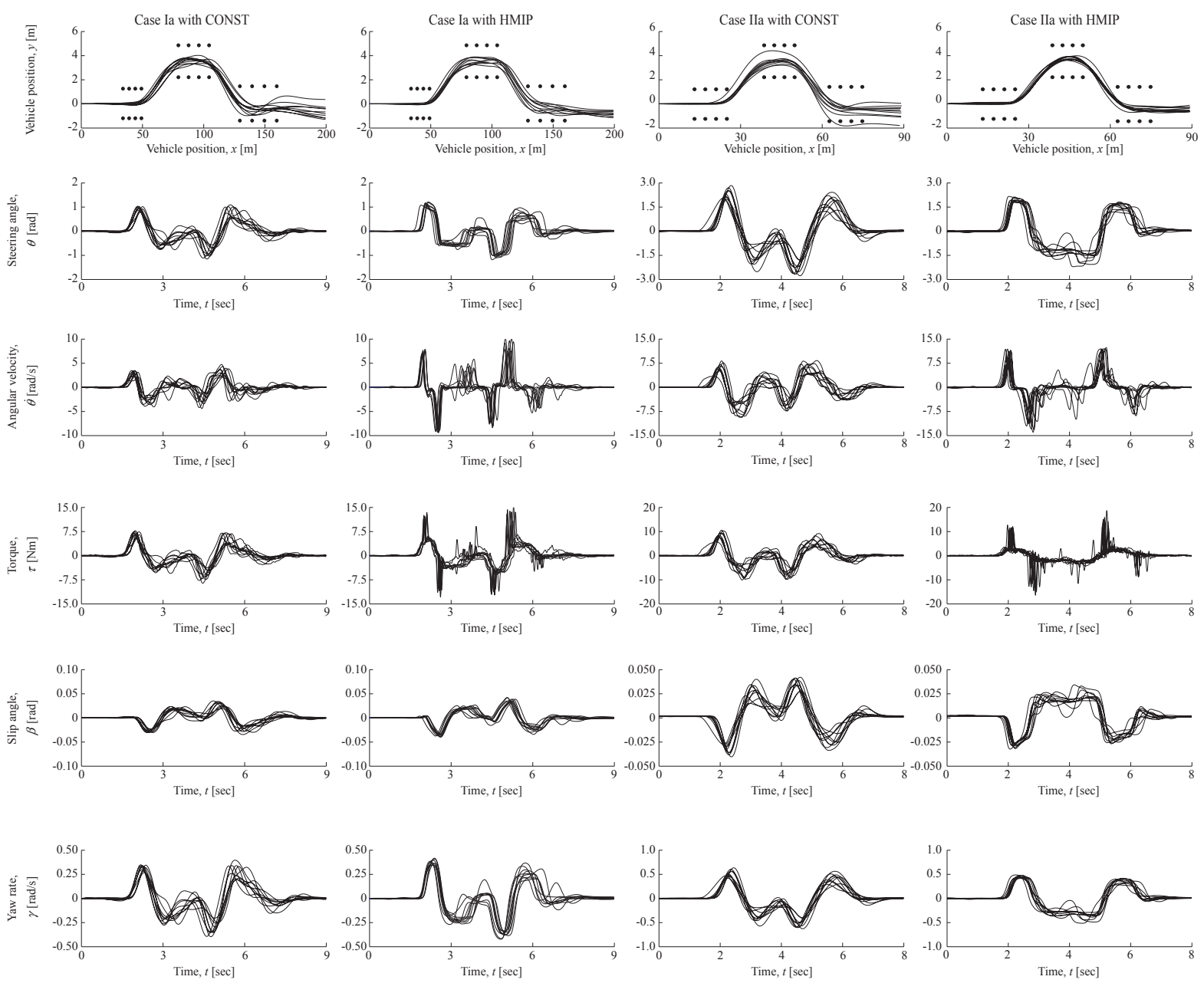

Fig. 11. Time histories of steering and vehicle behaviors in Case Ia and IIa (Sub. A).

suggest that the proposed control system can actively assist driver's steering motions by adjusting the damping coefficient of the human-steering system without any degradation in the driving performances for an emergency obstacle avoidance task under the DLC test.

\section{Operational Experiment II: DLC test using a 4-DOF driving simulator}

The effectiveness of the proposed control methodology was further examined using a 4-DOF driving simulator developed by Mazda motor corporation as shown in Fig. 13. Four subjects (Subs. D, E, G, and H) were participated in the experiment, and five sets were conducted for Case Ia and Case Ib.

The 4-DOF driving simulator is composed of a computer for managing the whole system, a movable cockpit, a vehicle motion simulator, a projector-screen system to monitor road images and a vehicle sound simulator. The cockpit has three degrees of freedom (roll, pitch and yaw) to provide a variety of driving feelings. A steering wheel and a torque sensor are attached to the rotating part of an electric motor, and the steering angle is measured by an encoder built into the motor. Vehicle motion is calculated from the operational signals for driving interface devices with a vehicle model, and the driving scene is projected to the screen placed in the cockpit. The vehicle sound simulator creates realistic sounds of engine and load noise according to the vehicle condition.

Fig. 14 shows the typical time histories of steering and vehicle behaviors for five sets of the experiments measured in each control system, in which the trajectories of the vehicle's center of gravity, the steering angle $\theta$, the angular velocity $\dot{\theta}$, the steering torque $\tau$, the slip angle $\beta$, the yaw rate $\gamma$ and the lateral acceleration $A_{y}$ are plotted from the top. The maximum lateral acceleration $A_{y_{-} \max }$ for the proposed control system tended to be lower. This argues that the proposed system improves not only obstacle avoidance capacity and vehicle controllability but also reduces driving load and erroneous operation caused by the lateral acceleration in steering for an emergency obstacle avoidance task.

The quantitative evaluation of steering and vehicle behaviors depending on the conditions was carried out by the two indices $E_{H}$ and $E_{V}$ given as

$$
\begin{aligned}
& E_{H}=\sqrt{T^{2}+T_{H}^{2}}, \\
& E_{V}=\sqrt{J^{2}+T_{V}^{2}},
\end{aligned}
$$

where the steering stability $T_{H}$ is the time when the steering angular velocity falls below $0.05 \mathrm{rad} / \mathrm{s}$, the vehicle stability $T_{V}$ 

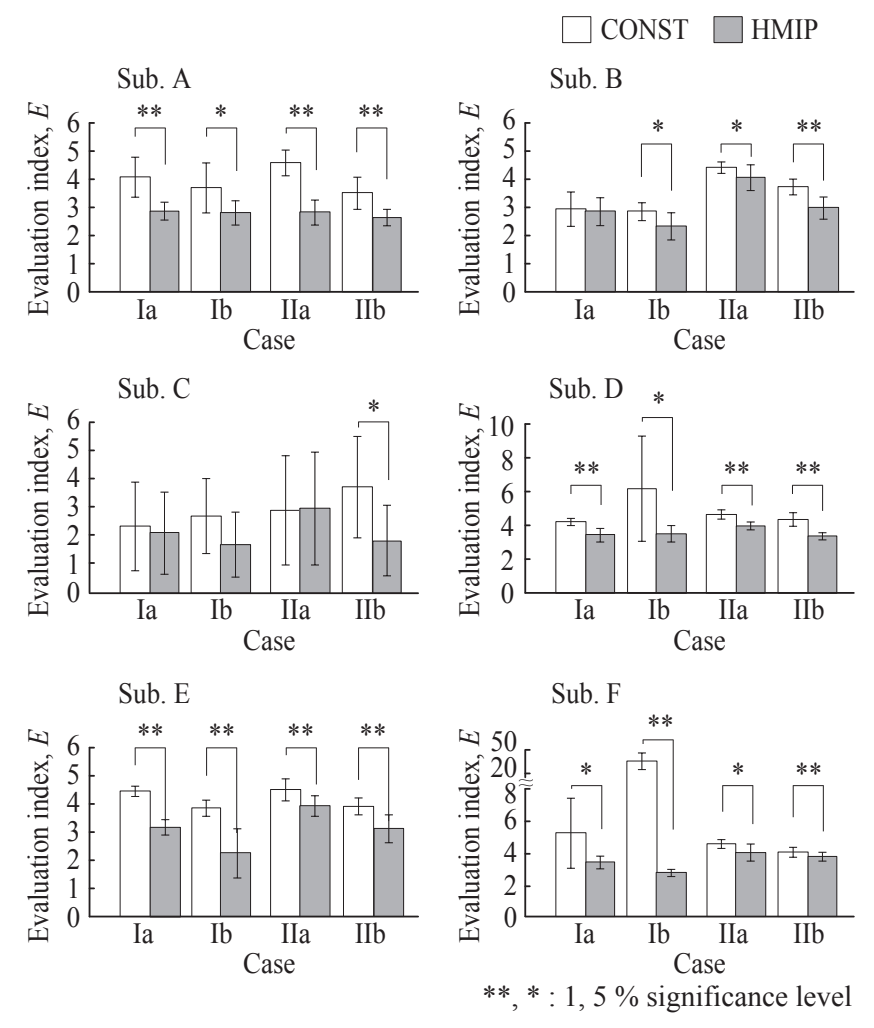

Fig. 12. Control performances of the driver-vehicle system for all subjects according to the specified test cases.

is the time when the yaw rate falls below $0.05 \mathrm{rad} / \mathrm{s}$, respectively. Lower values of $T_{H}$ and $T_{V}$ mean higher stability in the steering and vehicle behaviors after obstacle avoidances, and those of $E_{H}$ and $E_{V}$ mean better controllability. Fig. 15 shows the evaluation results of $E_{H}$ and $E_{V}$ for all subjects, and demonstrates that the values for HMIPS was lower compared to those for CONST, especially in Case Ib, although some amount of difference between individuals can be seen.

Finally, subjective evaluation of the proposed control methodology was done regarding the obstacle avoidance capacity, handling and stability by using a five-point rating method. Fig. 16 shows the mean values and standard deviations of each subjective factor for all subjects, and indicates that the proposed control system well contributed to improve the driving feels in addition to the driving performances.

Consequently the successful integration of HMIPS into a steering control system causes the betterment of steering performance and operational feeling in vehicle driving.

\section{CONCLUSion}

This paper reveled how the HMIP during steering operations by the arms changed in the limbs posture (steering angle) and muscle co-contraction level (steering torque), and proposed a HMIP-based adaptive steering control system in which the steering properties were regulated depending on the steering angle, torque and vehicle dynamics. A set of the DLC experiments were carried out for the several subjects using the two type of driving simulator that implemented the proposed steering control structure, and the effects of

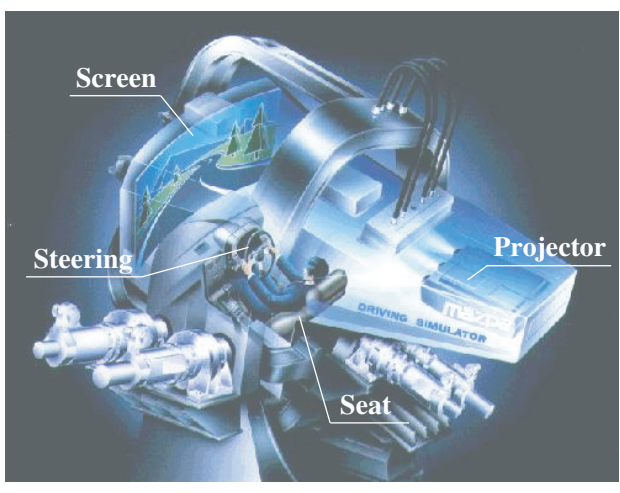

(a)

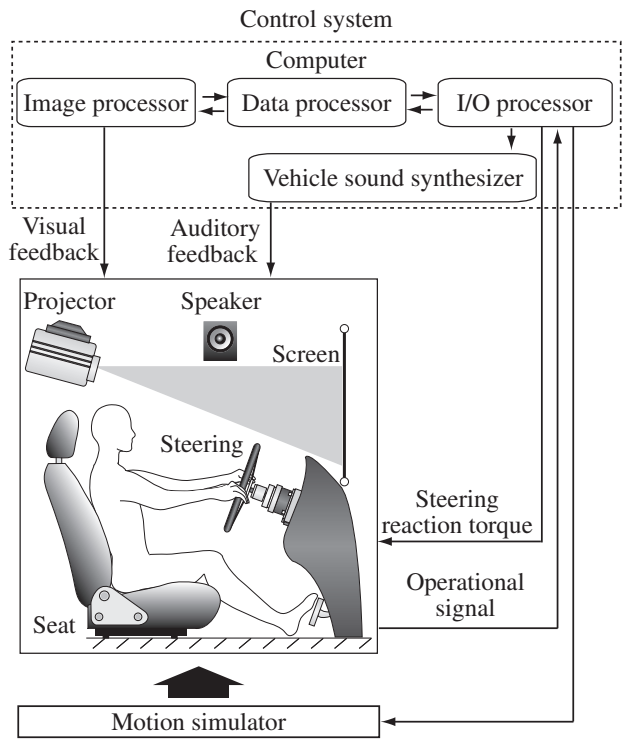

(b)

Fig. 13. A 4-DOF driving simulator for producing realistic translation and rotation of a vehicle.

regulating the damping coefficient of the driver-vehicle system was analyzed with the conventional control structure. The results demonstrated that the driving performance, such as the quickness and accuracy of steering and vehicle behaviors, could be successfully improved with an appropriate regulation function of the damping coefficient for the specified driving situation.

The subjects participated in this paper were limited to young male with little driving career, and thus the validations of the proposed steering control system should be carried out for other subjects with different backgrounds. Future research will discuss further improvements to handling in considerations of driver's individual differences, as well as design problem of the damping coefficient in consideration of other situations.

\section{Acknowledgment}

The authors should deeply thank Mr. Y. Kashiba for his large contribution to this research work.

\section{REFERENCES}

[1] D. Peter and R. Gehrad, "Electric power steering - The first step on the way to steer by wire," SAE Technical Paper, 1999-01-0401, 1999. 

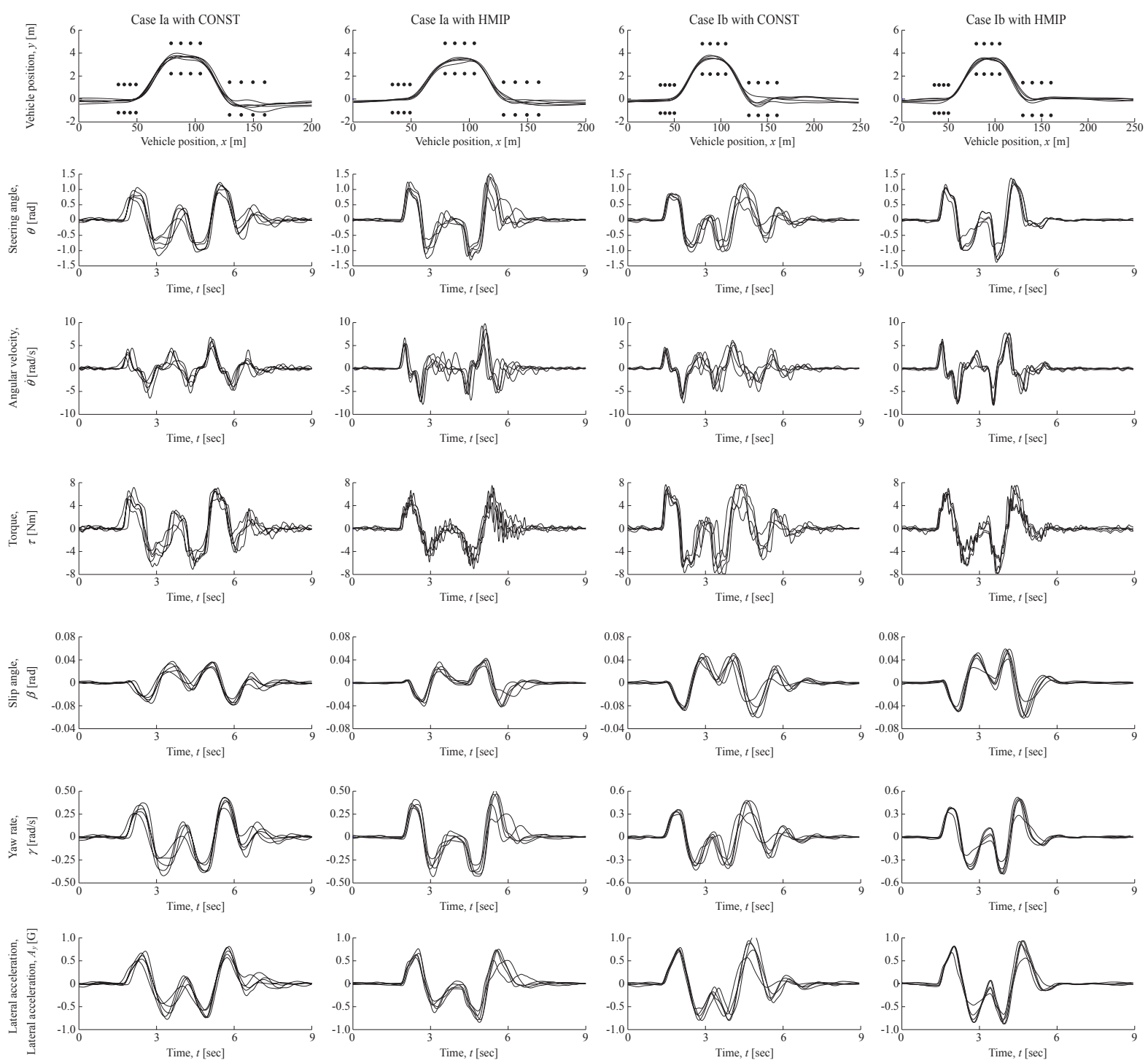

Fig. 14. Time histories of steering and vehicle behaviors in Case Ia and Case Ib using the 4-DOF driving simulator (Sub. G).

[2] Y. Yao, "Vehicle Steer-by-Wire System Control," SAE Technical Paper, 2006-01-1175, 2006.

[3] F. A. Mussa-Ivaldi, N. Hogan, and E. Bizzi, "Neural, mechanical and geometric factors subserving Arm Posture in Humans," J. of Neuroscience, vol. 5, no. 10, pp. 2732-2743, 1985.

[4] J. M. Dolan, M. B. Friendman, and M. L. Nagarka, "Dynamics and loaded impedance components in the maintenance of human arm posture," IEEE Trans. on Systems, Man and Cybernetics, vol. 23, no. 3, pp. 698-709, 1993.

[5] T. Tsuji, P. G. Morasso, K. Goto, and K. Ito, "Human hand impedance characteristics during maintained posture in multi-joint arm movements," Biological Cybernetics, vol. 72, pp. 475-485, 1995.

[6] F. Lacquaniti, M. Carrozzo, and N. A. Borghese, "Time-varying mechanical behavior of multijointed arm in man," Journal of Neurophysiology, vol. 69, pp. 1443-1464, 1993.

[7] H. Gomi and M. Kawato, "Equilibrium-point control hypothesis examined by measured arm stiffness during multijoint movement," Science, vol. 272, pp. 117-120, 1996.

[8] B. B. Litkouhi, A. Y. Lee, and D. B. Craig, "Estimator and controller design for lane track, a vision-based automatic vehicle steering system," in Proc. of the 32nd IEEE CDC, 1993, pp. 1868-1873.

[9] S. Amberkar, F. Bolourchi, J. Demerly, and S. Millsap, "A control system methodology for steer by wire systems," SAE Technical Paper, 200401-1106, 2004.

[10] P. Yih and J. C. Gerdes, "Modification of vehicle handling characteristics via steer-by-wire," IEEE Trans. on Control Systems Technology, vol. 13, issue 6, pp. 965-976, 2005.

[11] N. Bajcinca, R. Cortesão, and M. Hauschild, "Robust control for steerby-wire systems," Autonomous Robots, vol. 19, issue. 2, pp. 193-214, 2005.

[12] A.J. Pick and D.J. Cole, "Dynamic properties of a driver's arms holding a steering wheel," Proc. of the Institution of Mechanical Engineers, Part D: Journal of Automobile Engineering, vol. 221, pp. 1475-1486, 2007.

[13] A.J. Pick and D.J. Cole, "A mathematical model of driver steering control including neuromuscular dynamics," Trans. of the ASME, Journal of Dynamic Systems, Measurement, and Control, vol. 130, 031004, 2008.

[14] D.A. Abbink, M. Mulder, and E.R. Boer, "Haptic shared control: smoothly shifting control authority?" Cognitive Technology Work, vol. 14, pp.19-28, 2012.

[15] J. Venrooji, M. Mulder, D. A. Abbink, M. M. van Paassen, and F. C. T. van der Helm, "A new view on biodynamic feedthrough analysis: Unifying the effects on forces and positions," IEEE Trans. on Cybernetics, vol. 43, no. 1, pp. 129-141, Feb. 2013.

[16] R. Ikeura, H. Hoshino, D. Yokoi, Y. Kanehara, H. Hoshino, K. Mizutani, and H. Sawai, "A rating method for the vehicle steering based on the impedance of human arms," Trans. of Society of Automotive Engineers of Japan, vol. 37, no.4, pp. 33-38, 2006 (in Japanese).

[17] N. Hogan, "Impedance control: an approach to manipulation: Parts I, II, III," ASME Journal of Dynamic Systems, Measurement, and Control, vol. 107, no. 1, pp. 1-24, 1985.

[18] H. Kazerooni, "Human-robot interaction via the transfer of power and information signals," IEEE Trans. on Systems, Man, and Cybernetics, 


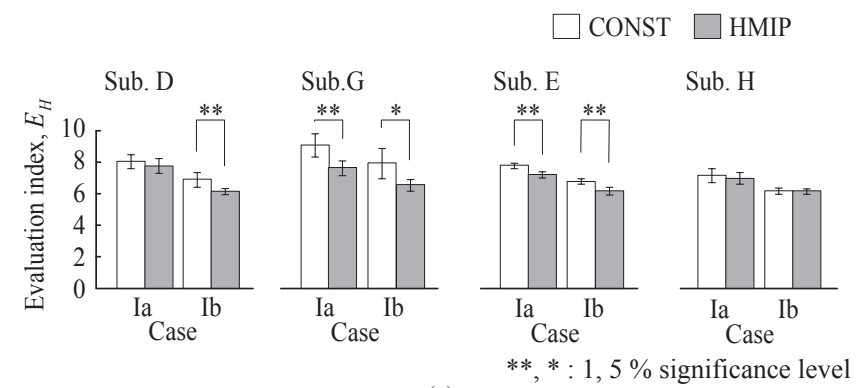

(a)
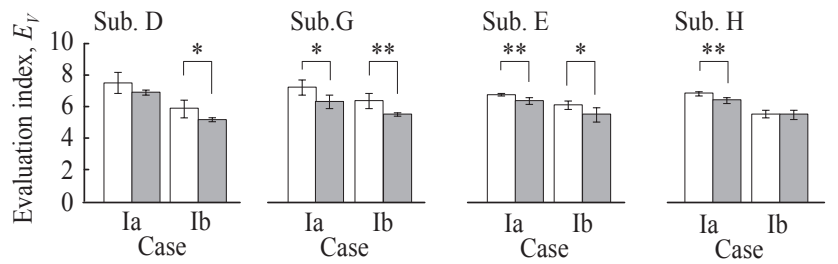

(b)

Fig. 15. Control performances of the driver-vehicle system for all subjects.

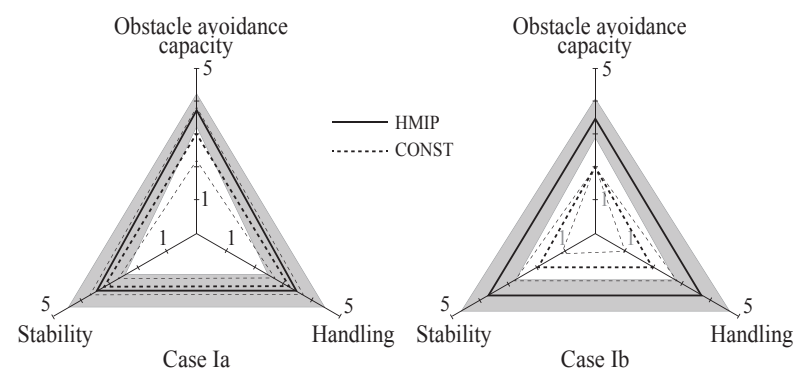

Fig. 16. Subjective evaluation results regarding obstacle avoidance capacity, handling and stability.

vol. 20, no. 2, pp. 450-463, 1990.

[19] K. Kosuge and N. Kazamura, "Control of a robot handling an object in cooperation with a human," in Proc. of the IEEE Int. Workshop on Robot and Human Communication, 1997, pp. 142-147.

[20] J. E. Colgate, "Robust impedance shaping telemanipulation," IEEE Trans. on Robotics and Automation, vol. 9, no. 4, pp. 374-384, 1993.

[21] R. Ikeura and H. Inooka, "Variable impedance control of a robot for cooperation with a human," in Proc. of the IEEE ICRA, 1995, pp. 30973102.

[22] O. M. Al-Jarrah and Y. F. Zheng, "Arm-manipulator coordination for load sharing using compliant control," in Proc. of the IEEE ICRA, 1996, pp. $1000-1005$.

[23] T. Tsumugiwa, R. Yokogawa, and K. Hara, "Variable impedance control based on estimation of human arm stiffness for human-robot cooperative calligraphic task," in Proc. of the IEEE ICRA, 2002, pp. 644-650.

[24] R. J. Adams, and B. Hannaford, "Stable haptic interaction with virtual environments," IEEE Trans. on Robotics and Automation, vol. 15, no. 3, pp. 465-474, June 1999.

[25] E. L. Faulring, K. M. Lynch, J. E. Colgate, and M. A. Peshkin, "Haptic display of constrained dynamic systems via admittance displays," IEEE Trans. on Robotics, vol. 23-1, pp. 101-111, Feb. 2007.

[26] T. Yoshikawa, Y. Yokokohji, T. Matsumoto, and X. Z. Zheng, "Display of feel for the manipulation of dynamic virtual objects," Trans. of the ASME, Journal of Dynamic Systems, Measurement, and Control, vol. 117-4, pp. 554-558, Dec. 1995.

[27] M. Abe, "Motion and control of vehicle," SANKAIDO PUBLISHING Co., Ltd, 2003 (in Japanese).

[28] S. Okawa and A. Honda, "Technical manual of the motion control of vehicle," SANKAIDO PUBLISHING Co., Ltd, 2006 (in Japanese).

[29] Society of automotive engineers of Japan, Inc., "Automotive Engineers Hand Book, Vol. 3, Test/Evaluation stitch," SEIKOSHA, 1991 (in Japanese). 